\title{
Polymorphism in the promoter region of the squalene synthase gene in different amaranth species
}

\author{
A.B. Shcherban*, A.I. Stasyuk, E.A. Salina \\ Institute of Cytology and Genetics SB RAS, Novosibirsk, Russia \\ *e-mail: atos@bionet.nsc.ru
}

Key words: amaranth, squalene, squalene synthase gene, promoter, polymorphism

Motivation and Aim: Grain amaranth is potentially an important source of squalene, a natural 30-carbon organic compound that has numerous commercial purposes such as lubrication and protection of the skin (in cosmetics), as an adjuvant in vaccines (in medicine), etc. According to previous reports, the squalene concentration in amaranth oil is many times higher than that in other plant oils and varies between different species of amaranth [1]. The squalene synthase (SQS) gene may play a significant role in regulation of squalene biosynthesis. However, structural polymorphism of this gene among amaranth species differing in squalene content have not yet been studied.

Methods and Algorithms: Based on the known coding sequence of the SQS gene from $A$. cruentus [2], we downloaded the corresponding nucleotide sequence of A. hypochondriacus (including the promoter region $\sim 1 \mathrm{~kb}$ long), using the highquality draft genome sequence of this species available at GoGe (id40120; (https:// genomevolution.org/coge/). The predicted amino acid SQS sequences from both species showed a high level of identity, especially, within the most conservative functional domains. We designed specific primers to the promoter region of the SQS gene of A. hypochondriacus and used them to amplify and sequence this region from 95 accessions related to 23 amaranth species. Multiple sequence alignments and the subsequent phylogenetic analysis were carried out using the ClustalW program and MEGA4 software. The putative cis- regulatory elements in the gene promoter were searched using database PlantPAN 2.0 (http://plantpan2.itps.ncku.edu.tw).

Results: A high level of interspecific polymorphism was revealed inside the promoter of the SQS gene. The most significant variation that could affect a probable transcriptional regulatory sites was found immediately upstream the putative TATA-box located at position -168 from ATG-codon. Based on the alignment of the nucleotide SQS promoter sequences the neighbor-joining tree was constructed.

Conclusion: The results obtained imply a major impact of the SQS promoter on the regulation of the gene expression and allow to proceed to the next stage, the study of association between the promoter polymorphism and the SQS expression patterns which in turn may determine production of squalene in different amaranth species.

\section{References}

1. He H.P. et al. (2002) Extraction and purification of squalene from Amaranthus grain. J. Agric. Food Chem. 50:368-372.

2. Park Y.-J. et al. (2016) Molecular cloning, expression and characterization of a squalene synthase gene from grain Amaranth (Amaranthus cruentus L.). Japan Agricultural Research Quarterly. 50(4):307-317. 